\title{
IOE: TOWARDS APPLICATION-SPECIFIC TECHNOLOGY SELECTION
}

\author{
Biswajit Paul $^{1}$, Gokul Chandra Biswas ${ }^{2}$, Habib F. Rashvand ${ }^{3}$ \\ ${ }^{1}$ Electrical and Electronic Engineering, Shahjalal University of Science and Technology, Sylhet, Bangladesh, ${ }^{2}$ Genetic \\ Engineering and Biotechnology, Shahjalal University of Science and Technology, Sylhet, Bangladesh, ${ }^{3}$ Advanced \\ Communication Systems, University of Warwick, Coventry, UK \\ NOTE: Corresponding author: Biswajit Paul, biswajit-eee@sust.edu
}

\begin{abstract}
Determining the suitability of any technology for an Internet of Everything (IoE) application is essential in the presence of diverse technologies and application requirements. Some of the IoE applications include smart metering, wearables, healthcare, remote monitoring, inventory management and industrial automation. Energy efficiency, scalability, security, low-cost deployment and network coverage are some of the requirements that vary from one application to another. Wireless technologies such as WiFi, ZigBee, Bluetooth, LTE, NB-IoT, LoRa and SigFox will play crucial roles in enabling these applications. Some of the technological features are transmission range, bandwidth, data rate, security schemes and infrastructure requirements. As there is no one-size-fits-all network solution available, the key is to understand the diverse requirements of different IoE applications and specific features offered by different IoE enabling technologies. Application-specific technology selection will ensure the best possible utilization of any technology and the quality of service requirements. An overview of network performance expectations from various IoE applications and enabling technologies, their features and potential applications are presented in this paper.
\end{abstract}

Keywords - IoE, LPWANs, M2M, MTC, network design, suitable technology selection, wireless technologies

\section{ABBREVIATIONS}

- 3GPP - 3rd Generation Partnership Project

- 8PSK - Eight Phase Shift Keying

- AMI - Advanced Metering Infrastructure

- BPSK - Binary Phase Shift Keying

- CAPEX - Capital Expenditure

- COPD - Chronic Obstructive Pulmonary Disease

- CSMA/CA - Carrier Sense Multiple Access with Collision Avoidance

- D2D - Device to Device

- DBPSK - Differential Binary Phase Shift Keying

- DMM - Disributed IP Mobility Management

- DSO - Distribution System Operators

- DSSS - Direct Sequence Spread Spectrum

- EC-GSM-IoT - Extended Coverage Global System for Mobile Communications for the Internet of Things

- eDRX - extended Discontinuous Reception

- eGPRS - enhanced General Packet Radio Service

- eMTC - enhanced Machine Type Communication

- EVs - Electric Vehicles
- FHSS - Frequency Hopping Spread Spectrum

- GFSK - Gaussian Frequency Shift Keying

- GMSK - Gaussian Minimum Shift Keying

- GW - Gateway

- HANs - Home Area Networks

- HCO - Healthcare Organization

- IC-IoE- Information-Centric IoE

- IIoT - Industrial Internet of Everything

- IoE - Internet of Everything

- IoT - Internet of Things

- IP - Internet Protocol

- ISM - Industrial, Scientific and Medical

- LoS - Line of Sight

- LPWANs - Low Power Wide Area Networks

- LR-WPAN - Low Rate Wireless Personal Area Network

- M2M - Machine to Machine

- MLANs - Meter Local Area Networks

- MMC - Massive Machine Communications

- mMTC - Massive Machine Type Communications 
- MN - Moving Networks

- MTC - Machine Type Communication

- MTDs - Machine Type Devices

- NANs - Neighborhood Area Networks

- NB-IoT - Narrowband Internet of Things

- NOMA - Non Orthogonal Multiple Access

- OPEX - Operational Expenditure

- OS - Operatig System

- PER - Packet Error Ratio

- PLC - Power Line Communication

- PMIPV6 - Proxy Mobile IPv6

- PSM - Power Saving Management

- PWPN - Power Wireless Private Network

- QoS - Quality of Service

- RF - Radio Frequency

- RPMA - Random Phase Multiple Access

- RSUs - Roadside Units

- SGs - Smart Grids

- SMs - Smart Meters

- SPHERE - Sensor Platform for Residential Environment

- UAV - Unmanned Aerial Vehicle

- UDN - Ultra-Dense Network

- UNB - Ultra-Narrowband

- URLLC - Ultra-Reliable Low Latency Communications

- V2I - Vehicle to Infrastructure

- V2N - Vehicle to Network

- V2P - Vehicle to Pedestrian

- V2V - Vehicle to Vehicle

- V2X - Vehicle to Everything

- WANs - Wide Area Networks

- WBANs - Wireless Body Area Networks

- WIA-PA - Wireless Networks for Industrial Automation for Process Automation

- WirelessHART - Wireless Highway Addressable Remote Transducer
- WISA - Wireless Interface for Sensors and Actuators

- WLAN - Wireless Local Area Network

- WPCN - Wireless Powered Communication Network

- WSNs - Wireless Sensor Networks

\section{INTRODUCTION}

The term 'Internet of Things' (IoT) refers to the network of physical objects or things embedded with electronics, software, sensors and network connectivity where information exchange takes place automatically [1]. The term IoE is preferred over IoT by many as IoE comprehensively addresses the connectivity of various technologies, processes and people while IoT addresses interconnectivity of physical objects, data inputs and outputs. Humans, monitoring sensors, healthcare equipment, sensorequipped automobiles etc. are considered in 'Everything' [2]. A significant increase in the number of deployed IoE devices can be observed in recent years as the IoE concept receives broader industry momentum. Some predictions on the IoE deployment scale [3], technology's market penetration [4] and estimated revenue generation [5] can be found in the literature. IoE promises ease of flow of information efficiently in a fast-paced world with various envisioned application types such as IoE devices from mobiles, smart home energy management systems, supporting disabled people, tracking human behaviour, underwater sensor networks, military affairs and autonomous cars. Agriculture, healthcare, environment, transport, industrial automation etc. are some of the potential IoE application domains. IoE will incorporate both humans and machines as suggested by some IoE applications where interaction with humans [6], places of residence [7], human nature [8], and environment [9] are observed.

Since IoE application requirements are diverse, network designs are often facilitated by differentiating Machine to Machine (M2M) networks from Machine Type Communication (MTC) networks. M2M communication includes the remote control of machines, monitoring, and collecting data from machines, whereas in MTC, typically, devices are small, inexpensive and can operate for an extended period without human intervention. M2M communication networks differentiate themselves from networks that relay traffic generated or consumed by humans in IoE. Examples of MTC are smart community, smart building, smart grid, smart water system etc. Network connectivity, communication protocols, middleware frameworks, etc. need careful consideration to support the massive number of devices. The heterogeneous nature of traffic such as static, intermittent, delay-sensitive, delay-tolerant, small or large packets and application-specific performance objectives can make the wireless network design more complicated and challenging. For example, the tolerable delay and an update frequency for the waste management application are 30 minutes and 1 hour, respectively. On the other hand, in- 
dustrial monitoring and supervision applications can tolerate delays in a range of milliseconds and have update frequencies in the range of seconds [5].

Cellular networks will play a major role in the IoE domain in supporting M2M communication networks. However, future cellular standards will require optimizing the access network for both broadband and M2M communications to meet varying design challenges. In contrast to broadband networks, large-scale deployment of inexpensive low-complexity devices, smaller payload sizes with non-uniform traffic density, energy efficiency, extended network coverage are required for M2M networks [4]. Some enhancements have been proposed in the 3rd Generation Partnership Project (3GPP) to efficiently support M2M applications in 2G, 3G, LTE Cat-1 and higher networks. Extended Coverage Global System for Mobile Communications for the Internet of Things (ECGSM-IoT) and Narrowband Internet of Things (NB-IoT) are cellular-based IoE enabling technologies. Besides the cellular-based technologies, short-range technologies such as Bluetooth, ZigBee and Wi-Fi, and non-cellularbased technologies such as LoRa and Sigfox will play vital roles to meet the huge connectivity demand placed by MTC networks.

Application-specific technology selection requires careful preparation such as analyzing energy efficiency, latency, reliability, reliability, scalability and security requirements. Video surveillance, a smart city application, cannot tolerate large delays compared to other smart city applications such as structural health monitoring and waste management. Video surveillance is an example of a high data-rate application while structural health monitoring and waste management are low data-rate applications. Some industrial applications such as closed-loop control/ interlocking and control require low data rates while delays in milliseconds are tolerated with a high update frequency. Average message sizes and average message transaction rates also vary from one application to another. For example, average message sizes and average message transaction rates are 20 bytes and $1.67 \times 10^{-3} / \mathrm{s}$ respectively for a typical home security application, and 1 bytes and $3.33 \times 10^{-2} / \mathrm{s}$ respectively for roadway signs. Some of the smart city applications such as road safety in urban/highways and most of the industrial applications such as factory automation/packaging machines are latency-critical IoE applications with high-reliability requirements.

Operating frequency, bandwidth, transmission range and data rate are some of the technological features of any technology. LoRa and Sigfox operate in the unlicensed Industrial, Scientific and Medical (ISM) spectrum band while EC-GSM-IoT and NB-IoT operate in licensed spectrum bands. Bluetooth and WiFi are two short-range technologies having transmission ranges of $50 \mathrm{~m}$ and 100 $m$ respectively. Although highly dependent on communication environments, some researchers reported that LoRa and SigFox can achieve approximately $15 \mathrm{~km}$ and $20 \mathrm{~km}$ transmission ranges respectively. Cellular-based technologies such as NB-IoT, EC-GSM-IoT, eMTC can also achieve a long transmission range. Bluetooth, WiFi, NBIoT, EC-GSM-IoT, eMTC have higher channel bandwidths compared to LoRa and SigFox. Channel bandwidths for Bluetooth and WiFi are $2 \mathrm{MHz}$ and $22 \mathrm{MHz}$ respectively while the channel bandwidth of SigFox is $100 \mathrm{~Hz}$ only. Bluetooth, WiFi, NB-IoT, EC-GSM-IoT, eMTC can support high data-rate applications while LoRa, SigFox support low data-rate applications.

The knowledge of application requirements and technological features of any technology can help us determine the suitability of that technology for a particular application. For example, WiFi, Bluetooth, NB-IoT will fit well in high throughput applications while LoRa and SigFox will not fit such applications. However, WiFi and Bluetooth are suitable for applications that require a small network coverage. On the other hand, LoRa and SigFox can provide larger network coverage. These observations can be collectively applied towards application-specific technology selection. Our concept is illustrated in Fig.1. Fig.2 shows the speed at which the IoE market is growing [10]. This paper is organized as follows: various IoE application requirements and network design constraints are discussed in Section 2, some IoE enabling technologies and features are discussed in Section 3, various IoE applications and enabling technologies are discussed in Section 4 and conclusions are drawn in Section 5.

\section{IOE APPLICATION REQUIREMENTS AND NETWORK DESIGN CONSTRAINTS}

The diverse nature of IoE application scenarios may have a diverse set of requirements. Some of the requirements could be application-specific while others fall into general expectations. Some requirements arise from the typical IoE devices and business objectives while others are relevant to networks. As an example, average message transaction rates and average message sizes are shown in Table 1. Long battery life, support for the massive number of devices, extended coverage, low device cost, low deployment cost, security and privacy etc. are some of the key requirements for some applications. Network scalability, throughput, cell capacity, interference and delay are important considerations for other IoE applications.

\subsection{Energy efficiency}

The most important issue in IoE networks is probably energy efficiency [5]. Since the end devices are operated by irreplaceable batteries and the network is expected to be functional for a long time without human intervention in applications such as fire warning and pipeline inspection [11], battery energy should be utilized most efficiently. A battery life span expectation of 10 years for network operation is reported in [5]. A significant amount of energy is consumed in packet transmission and reception processes compared to other processes. The author in [12] discussed the requirement of delicate balancing between 
Application Requirements

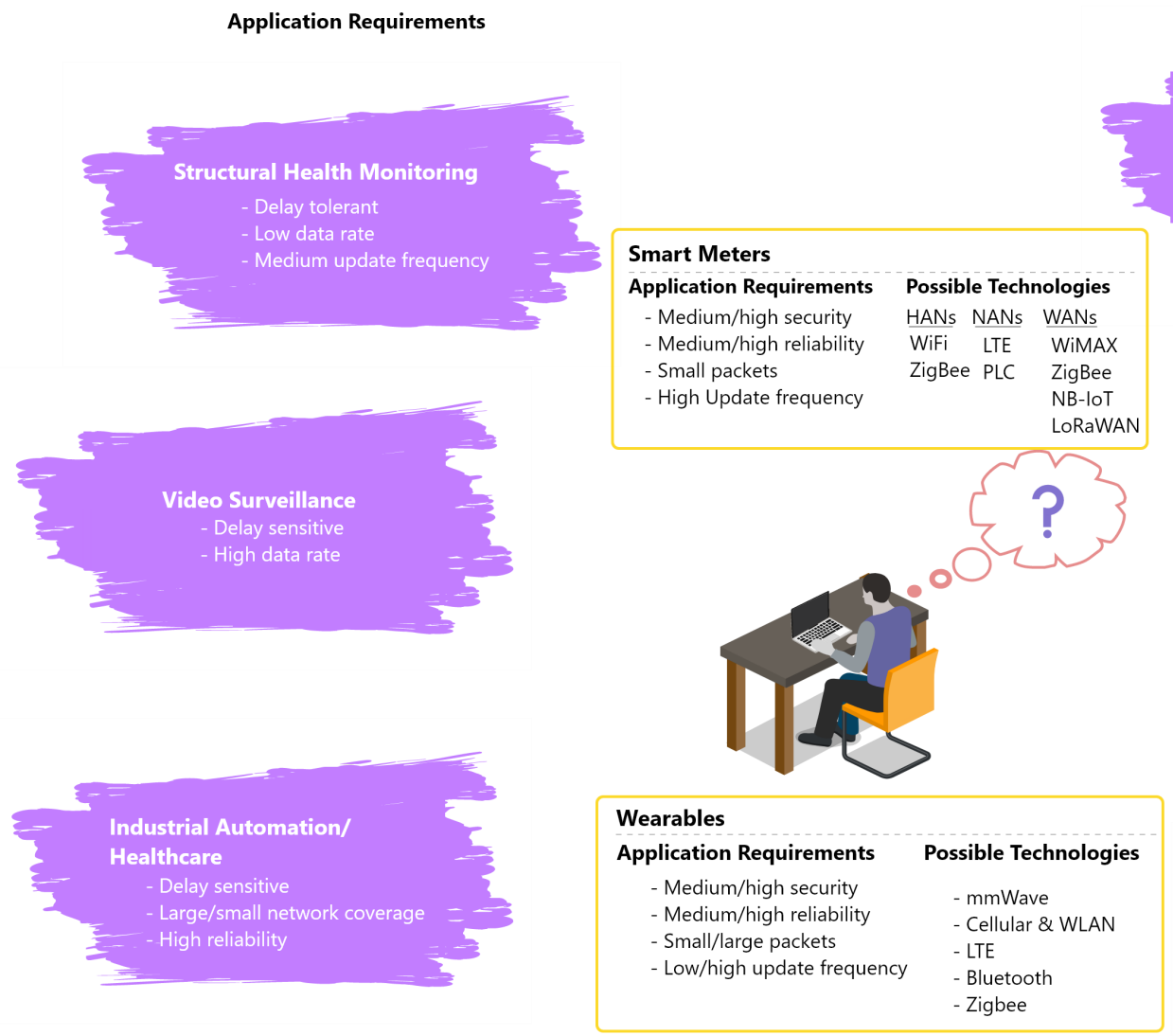

Technology Features
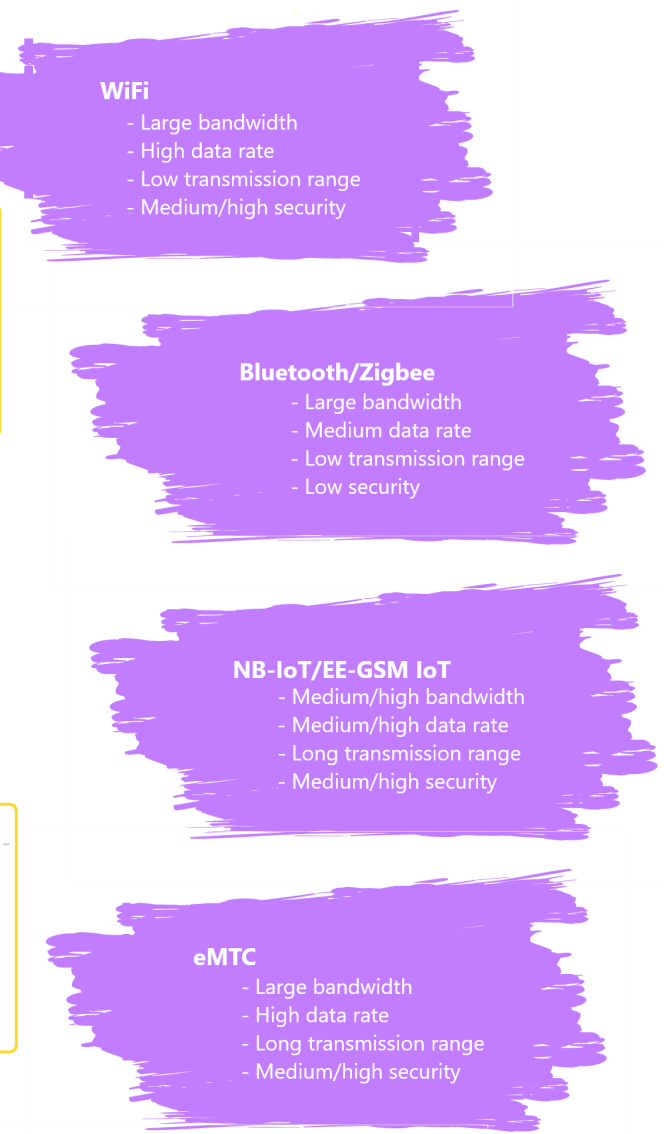
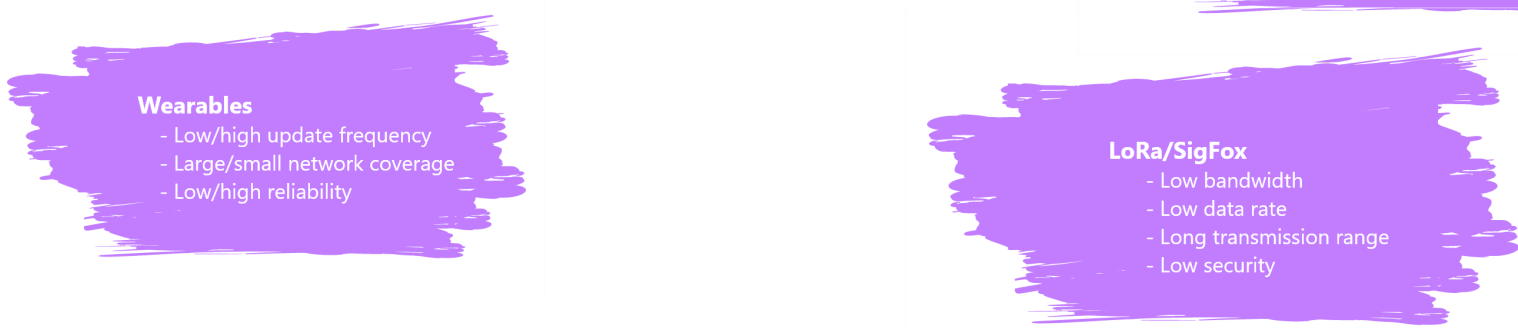

Fig. 1 - Application-specific technology selection

the number of packet transmissions and network lifetime. However, in some applications such as wearables devices where a significant volume of data may need to be processed, the circuit power consumption is often comparable to the transmit power [13].

Energy efficiency issues will have to be addressed through the design of hardware, software or MAC protocols, suitable routing scheme, efficient energy management system and energy harvesting. The multi-hop routing in [14] was found to be more energy-efficient than singlehop routing in LoRa networks while ensuring high network connectivity, low computational complexity for end nodes and addressing dynamic node distribution scenarios. On the other hand, the routing algorithm in [15] combined different energy harvesting techniques to improve the network lifetime and Quality of Service (QoS) under variable traffic load and energy availability conditions. Wireless power transfer enables the IoE nodes to collect energy from the Radio Frequency (RF) of the surrounding transmitters [16]. For Unmanned Aerial Vehicle (UAV) applications, UAV swarms can have a relatively good channel state to complete wireless power transfer as the probability of finding a Line of Sight (LoS) link is high [11]. Cognitive radio and Non-Orthogonal Multiple Access (NOMA) are candidate technologies for $5 \mathrm{G}$ networks for improving network spectral efficiency and scalability and the authors in [17] introduced a resource management framework for cognitive IoE networks with RF energy harvesting. In a Wireless Powered Communication Network (WPCN), multiple energy-limited devices first harvest energy in the downlink and then transmit information in the uplink. Although NOMA has been proposed to improve the system spectral efficiency in $5 \mathrm{G}$ networks, the authors in [13] found that NOMA-based WPCN not only consumes more energy but also is less spectrally efficient than TDMA-based WPCN. 


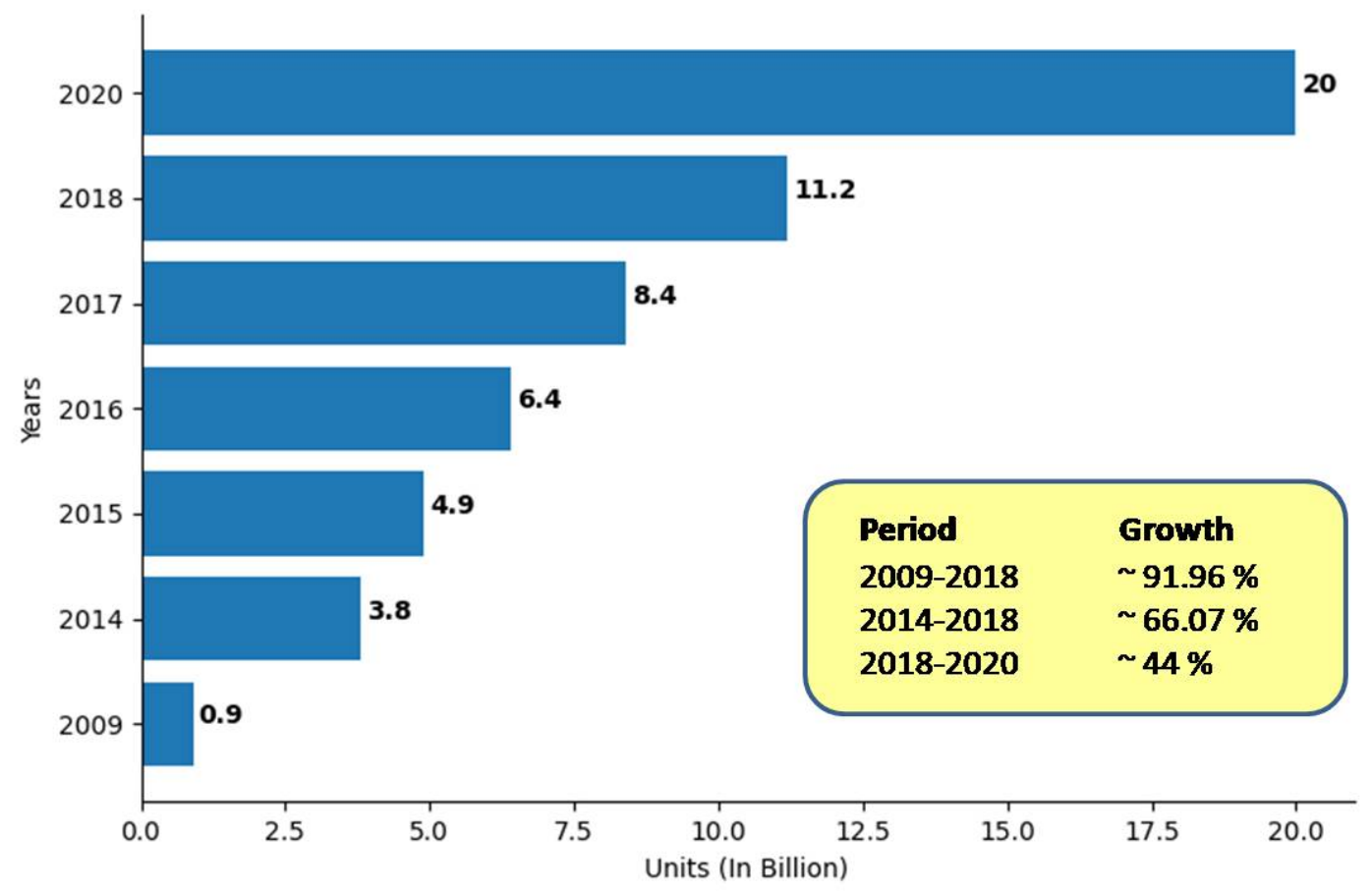

Fig. 2 - Growth of IoE devices

\subsection{Network coverage}

The requirement for extended network coverage is another key driving force for the introduction of LPWAN technologies. Extended network coverage will be required for some of the IoE applications; for example, smart meters located in the basement of buildings, behind a concrete wall or inside elevators will require an enhanced link budget. Also, the wireless coverage of UAVs for IoE should be extended rapidly and effectively in disaster-affected areas [18]. UAV-aided networks can establish wireless interconnections quickly, which is necessary for achieving larger wireless coverage. Multi-hop Device to Device (D2D) communications can be utilized to achieve larger coverage for UAVs [18]. Link budget and design parameters can be exploited to increase network coverage. The authors in [19] found that NB-IoT $882 \mathrm{MHz}$ and LoRaWAN can increase coverage by up to $398 \%$ and $142 \%$ respectively with a $10 \%$ improvement in receiver sensitivity. They also found that RPMA, NB-IoT and LTE$M$ incurs at least $9 \mathrm{~dB}$ additional path loss relative to Sigfox and LoRaWAN.

\subsection{Security and privacy}

Security incidents weaken the confidence in the IoE paradigm, hindering its widespread implementation. The disclosure of private and confidential information causes various privacy violations and business disruptions. However, the most significant danger remains the
Table 1 - Average message transaction rate and average message size for different IoE applications

\begin{tabular}{|l|l|l|}
\hline Application & $\begin{array}{l}\text { Average Message } \\
\text { Transaction Rate } \\
\left(s^{-1}\right)\end{array}$ & $\begin{array}{l}\text { Average } \\
\text { Message } \\
\text { Size (bytes) }\end{array}$ \\
\hline $\begin{array}{l}\text { Roadway } \\
\text { Signs }\end{array}$ & $3.33 \times 10^{-2}$ & 1 \\
\hline $\begin{array}{l}\text { Traffic Lights } \\
\text { or Traffic } \\
\text { Sensors }\end{array}$ & $1.67 \times 10^{-2}$ & 1 \\
\hline $\begin{array}{l}\text { House } \\
\text { Appliances }\end{array}$ & $1.16 \times 10^{-5}$ & 8 \\
\hline $\begin{array}{l}\text { Credit } \\
\text { Machine } \\
\text { in a Shop }\end{array}$ & $5.56 \times 10^{-4}$ & 24 \\
\hline $\begin{array}{l}\text { Home } \\
\text { Security }\end{array}$ & $1.67 \times 10^{-3}$ & 20 \\
\hline $\begin{array}{l}\text { Process } \\
\text { Automation }\end{array}$ & 0.2 to 10 & 40 to 100 \\
\hline Smart Grids & 10 to 100 & 80 to 1000 \\
\hline $\begin{array}{l}\text { Road Safety } \\
\text { Highway }\end{array}$ & 10 & $\leq 500$ \\
\hline $\begin{array}{l}\text { Traffic } \\
\text { Efficiency }\end{array}$ & 1 & $1 \mathrm{~K}$ \\
\hline $\begin{array}{l}\text { Urban } \\
\text { Intersection }\end{array}$ & 1 & $1 \mathrm{M} /$ car \\
\hline
\end{tabular}


threat to people's lives and wellbeing from IoE devices' exposure. Security risks in a healthcare setting, management of traffic lights/connected vehicles may cause accidents leading to fatalities besides causing havoc and increasing pollution [20]. The substantial difference between standard and IoE networks is the resourcefulness of the end devices. In contrast to traditional networks with overflowing resources, IoE devices mostly operate on low power, limited memory, limited computing ability and storage facility. Thus, a balance is required between security and resources as limited resources may restrict enabling technologies to lightweight security algorithms and protocols [21]. Besides, the IoE ecosystem faces diverse data formats and contents due to different application functionalities and the lack of a standard Operating System (OS). They are prone to generic threats such as hardware vulnerabilities, vulnerabilities of social engineering, DoS/ DDoS attacks [21]. Architecture layerwise threats may include eavesdropping, node cloning in the physical layer; unauthorized access, replication of nodes and injection of fake devices in the network layer etc. [21]. Research efforts are made to improve security in IoE networks. Security threats at different layers such as the sensing layer, network layer, middleware layer, gateways and application layer are presented in [22]. The authors in [22] also discussed existing and upcoming solutions to IoE security threats including blockchain, fog computing, edge computing and machine learning. Adoption of Distributed IP Mobility Management (DMM) for 5G networks and affiliated applications is highly predicted [23]. The flat architecture of DMM harmonizes well with $5 \mathrm{G}$ networks while overcoming the critical shortcomings of the centralized mobility management technologies such as Mobile IPv6 and Proxy Mobile IPv6 (PMIPv6) [24]. Protecting transmitted data traffic between user mobile devices and their in-home IoT appliances is of paramount importance as the data traffic may include users' sensitive and critical private information. The authors in [24] focused on secure route optimization to enable direct communication between end devices securely while minimizing the possibility of information leakage during data transmission.

\subsection{Network scalability}

Network scalability will be a key consideration as soon as the market gets bigger. IoE networks will have to support the inclusion of many new heterogeneous devices or exclusion of old devices to sustain market demand in the long run. Applications and functions for the interest of end users without compromising the quality and provision of existing services will have to be addressed too which in turn will put a constraint on network capacity. Network scalability, throughput and/or cell capacity issues have been studied in [25-29]. Transceivers are assumed to undergo high levels of cross and self-technology interference from heterogeneous environments of various wireless technologies and the massive number of IoE devices as LPWAN technologies remain their operations in unlicensed spectrums. Severe interference can potentially degrade network performance and service quality. A high level of interference will increase the Packet Error Ratio (PER) resulting in a loss of reliability. A high number of packet retransmissions might be required under these circumstances.

\subsection{Reliability}

Reliability is imperative for the safety-critical or missioncritical nature of the IoE applications. The diverse nature of technical requirements for different IoE networks poses a lot of challenges and for some applications, IoE networks are required to simultaneously support high reliability, low latency and massive connectivity [30]. Stringent transmission reliability is required for some applications such as industrial automation, Vehicle to Everything (V2X) networks, and smart grids [30]. Malfunctions of IoE devices, failure to capture critical data, network outage and data loss may result in catastrophic effects, such as mission failure, financial loss, and harm to people and environments [31]. The heterogeneous nature of IoE devices and networks requires diverse reliability protocols. Reliable architecture, operation and application development must address errors in the hardware, the software, interactions with the physical environment, and interactions with the human users [32]. The authors in [33] explored the reliability of the NB-IoT network in intelligent systems.

\subsection{Delay}

In the context of new $5 \mathrm{G}$ use cases, IoE applications have been categorized into two classes: massive Machine Type Communications (mMTC) and Ultra-Reliable Low Latency Communications (URLLC) [34]. mMTC applications will have demands for high network capacity, lowcost end devices and longer battery lifetime. On the other hand, mission-critical applications will rely on URLLC and will demand uninterrupted service with the huge volume of data exchange. M2M communication is widely utilized in a vast number of Industrial Internet of Everything (IIoE) applications. M2M communications in IIoE can be categorized as delay-sensitive and delay-tolerant. The control system in smart manufacturing lines monitors the condition of the manufacturing lines and makes real-time decisions. However, Machine Type Devices (MTDs) such as temperature and humidity sensors in manufacturing factories can tolerate a large delay. The coexistence of delay-sensitive MTDs and delay-tolerate MTDs requires clustering for efficient provisioning of heterogeneous delay requirements [35].

The contention over the limited network radio resources will increase, leading to network congestion with the increasing number of devices. Providing delay-aware channel access in cellular networks is essential for many IoE applications. Node clustering and data aggregation can 
play important roles in meeting the various service quality requirements of diverse applications [36]. In this context, a two-hop NOMA-enabled data aggregation architecture was proposed in [36] for massive cellular IoE applications. Moreover, a delay of no more than a few milliseconds is expected in biomedical applications. The authors in [37] discussed task offloading in wireless networks to save energy for devices and reduce the delay of processing tasks in IoE networks. A significant amount of medical data traffic will be produced with extensive use of IoEbased Wireless Body Area Networks (WBANs), leading to an imperative requirement for radio resource management with high utilization efficiency. It will be necessary to offer a priority-based transmission order to guarantee varying medical-grade QoS requirements [38].

\subsection{Network deployment cost}

Facilitating profitable business cases for IoE requires low device and network deployment costs. A modulo cost of less than $\$ 5$ is the current industrial target. Capital Expenditure (CAPEX) and Operational Expenditure (OPEX) should be kept at a minimum cost in the pursuit of achieving massive IoE applications and ensuring network connectivity [5]. With the non-uniform distributions of both the applications and humans with sensor devices in Information-Centric IoE (IC-IOE) networks, the information in the urban regions will be redundant and timely information collection in some regions will be challenging. Arranging plenty of static sensor devices will incur unrealistically huge costs for the IC-IoEs [39]. The authors in [40] focused on the design for jointly optimizing downlink and uplink operations to reduce costs in cellular-based IoE networks which provide connections to a massive number of IoE equipment following random access. Cost reduction in LoRa, Sigfox, and NB-IoT networks is also a vital issue, as they too are expected to connect a massive number of IoE equipment [40].

\section{IOE ENABLING TECHNOLOGIES}

D2D communications, Massive Machine Communications (MMC), Moving Networks (MN), Ultra-Dense Networks (UDN) and ultra-reliable networks are expected to be supported by $5 \mathrm{G}$ networks, while MMC forms the the basis of IoE $[41,42]$. Low Power Wide Area Networks (LPWANs) are suitable for massive IoE applications and typical applications include logistics, utilities, smart cities, consumer electronics, smart buildings, environment, agriculture and industry. LoRa, Sigfox, Ingenu, Random Phase Multiple Access (RPMA), DASH-7 and Weightless are some potential LPWAN technologies. Some of the traditional solutions like Bluetooth, Wi-Fi, ZigBee, WLAN, $\mathrm{Z}$ wave, GSM, LTE can provide wireless connections of the IoE devices in the network. However, these solutions demand high cost, high energy consumption and high complexity. While some of these technologies can support high bandwidth applications, they are unable to provide a larger communication range. IEEE working group 802.11ah enhanced communication development resulting in Bluetooth Low Energy 4.0, ZigBee and Wi-Fi/IEEE802.11 to support short-range communication for MTC [5]. On the other hand, EC-GSM-IoT, NB-IoT, LTE Cat-M1 are cellular-based LPWAN technologies that are intended to address the different IoE application requirements such as long-range, low power consumption, high bandwidth etc. Brief descriptions of some technologies are provided in the following subsections [43].

\subsection{Non-cellular-based LPWAN technologies}

LoRa: LoRa performs signal modulation in sub-GHz ISM bands using a spread spectrum technique which spreads a narrowband input signal over a wider channel bandwidth [44]. LoRa networks can utilize different data rates ranging from $300 \mathrm{bps}$ to a maximum of $50 \mathrm{kbps}$ and various transmission ranges with different spreading factors. The topology of LoRa networks is star-to-star where end devices communicate with a LoRa Gateway (GW) directly in single-hop using an ALOHA medium access scheme and to combat interference it relies on Frequency Hopping Spread Spectrum (FHSS) [5]. The technology utilizes different channel bandwidths such as $7.8 \mathrm{kHz}, 10.4 \mathrm{kHz}, 15.6$ $\mathrm{kHz}, 31.2 \mathrm{kHz}, 41.7 \mathrm{kHz}, 62.5 \mathrm{kHz}, 125 \mathrm{kHz}, 250 \mathrm{kHz}$ and $500 \mathrm{kHz}$. LoRaWAN adds a network layer to address network congestion between end devices and central nodes. $868 \mathrm{MHz}$ ISM bands in Europe and $915 \mathrm{MHz}$ bands in North America are used for network operation.

Sigfox: Sigfox utilizes Ultra-Narrowband (UNB) to offer complete end-to-end connectivity. Base stations in Sigfox are configured with cognitive software-defined radios while IP-based network infrastructure is utilized to connect them with backend servers [44]. End devices utilize a Binary Phase Shift Keying (BPSK) modulation scheme in an ultra-narrowband of $100 \mathrm{~Hz}$ sub-GHz ISM band carrier to connect themselves to the BS. SigFox operates in different frequency bands such as $868 \mathrm{MHz}$ and $915 \mathrm{MHz}$. Gaussian Frequency Shift Keying (GFSK) for downlink and Differential Binary Phase Shift Keying (DBPSK) for uplink transmission are used. The maximum packet size of 12 bytes and the maximum throughput of $100 \mathrm{bps}$ limit the number of use cases [44].

\subsection{Cellular-based LPWAN technologies}

Enhanced Machine Type Communication (eMTC): eMTC also known as LTE Cat-M1 or Cat-M is an enhancement for LTE networks to support MTC applications. This technology was introduced to reduce modem complexity, cost and power consumption while extending coverage [5]. The use of $20 \mathrm{dBm}$ power classes in Cat-M1 enables integration of power amplifiers and through avoiding a dedicated power amplifier achieves a lower device cost. A maximum coupling loss of $155.7 \mathrm{~dB}$ can be achieved with eMTC which marks an improvement of $15 \mathrm{~dB}$ over LTE base-line of $140.7 \mathrm{~dB}$. Utilizing Power Saving Man- 
agement (PSM) and extended Discontinuous Reception (eDRX) like power-saving mechanisms, a long battery life of approximately 10 years for Cat-M1 devices is achieved while using a 5 Watt-Hour battery system.

Narrowband-Internet of Things (NB-IoT): 3GPP Release13 specification introduced the cellular LPWAN technology NB-IoT, also known as LTE Cat-NB1. Allowing a small fraction of network resources, NB-IoT can coexist with legacy GSM, GPRS and LTE technologies. Cat-NB1 supports a minimum system bandwidth of $180 \mathrm{kHz}$ which allows a GSM operator to replace one GSM carrier of 200 $\mathrm{kHz}$. The maximum data rates are $66 \mathrm{kbps}$ and 16.9 kbps for multi-tone and single-tone uplink transmission respectively. In the case of a downlink transmission, the maximum data rates are $32 \mathrm{kbps}$ and $34 \mathrm{kbps}$ for in-band scenarios and standalone deployment respectively. NBIoT is seen as a promising technology to meet the huge traffic arising from various IoE applications making it an essential block for the $5 \mathrm{G}$ radio network.

Extended Coverage GSM for the Internet of Things (ECGSM-IoT): EC-GSM-IoT is based on enhanced General Packet Radio Services (eGPRS), introduced by 3GPP standardization in its Release-13 specification. Extended coverage and long employment duration are achieved through the upgradation of GSM networks. Utilizing eDRX, an efficient battery lifetime of 10 years can be achieved. $20 \mathrm{~dB}$ coverage extension is achieved with ECGSM-IoT compared to legacy GPRS networks. EC-GSMIoT can utilize two different modulation techniques: Eight Phase Shift Keying (8PSK) and Gaussian Minimum Shift Keying (GMSK). EC-GSM-IoT would enable the existing GSM networks to support massive IoE application deployment.

\subsection{Short range technologies}

Bluetooth: Bluetooth was designed for short-range adhoc communication between devices operating in the 2.4 GHz ISM bands and can support data rates in low Mbps. Bluetooth 4.0 improves power consumption and the recent amendment to the standard uses 40 channels with a width of $2 \mathrm{MHz}$ channel spacing. The technology uses GFSK for modulation, and FHSS to combat interference and multipath fading. Increased interest in developing the architecture for mesh networking can overcome the major drawback of Bluetooth which is a one-to-one communication between only two devices at a time.

IEEE 802.15.4 and ZigBee: IEEE 802.15.4 is the de facto standard for Low Rate Wireless Personal Area Networks (LR-WPAN). Network operation is performed in either $868 \mathrm{MHZ}$ or $914 \mathrm{MHz}$ or $2.4 \mathrm{GHz}$ band. Direct Sequence Spread Spectrum (DSSS) is used as the modulation scheme in IEEE 802.15.4. The maximum supported data rate is $250 \mathrm{kbps}$. A network layer on top of IEEE 802.15.4 physical and data link layer by ZigBee. ZigBee uses Carrier Sense Multiple Access with Collision Avoidance (CSMA/CA) for channel access and can support star, mesh, cluster tree topologies.
Wi-Fi: Wi-Fi is Wireless Local Area Network (WLAN) technology that belongs to the IEEE 802.11 standard series. It operates within $5 \mathrm{GHz}$ and $2.4 \mathrm{GHz}$ ISM spectrum bands. This technology provides high throughput connectivity between devices located nearby. Low-power Wi-Fi, which is also called IEEE 802.11ah is intended to serve a massive number of nodes distributed in a larger coverage area while consuming less power. The new standard targets approximately 100's of milliwatts of energy consumption for end devices and a data rate up to $347 \mathrm{Mbps}$ which would enable it to be used in different IoE applications such as parking metering, autonomous lightning, smart security etc.

Some of the important features of IoE enabling technologies are summarized in Table $2[5,45]$.

\section{IOE APPLICATIONS AND ENABLING TECHNOLOGIES}

The communication range of $\mathrm{Wi}-\mathrm{Fi} /$ Bluetooth is much smaller than other IoE technologies and therefore limiting the possible IoE use cases. Some potential IoE applications of Bluetooth and Wi-Fi can be found in [46, 47]. Personal activity, local object tracking, hospital asset tracking and point of sale could be some of the possible application scenarios of $\mathrm{Wi}-\mathrm{Fi} /$ Bluetooth. Some of the conceivable applications for ZigBee are waste management systems, warehouse logistics, home automation $[4,48]$. Wi-Fi/Bluetooth/ZigBee are suitable candidates for short-range high throughput applications while Wi$\mathrm{Fi} /$ Bluetooth can be also used for applications that require low latency and high reliability.

The well-established global ecosystem is a distinct advantage for cellular-based IoE enabling technologies. NBIoT, eMTC, EC-GSM-IoT are more likely to lead the high throughput/low latency applications market. They would also be able to scale up/scale down the network capacity according to market demands. However, spectrum sharing for IoE applications in the cellular domain is a challenging issue as it can hamper the existing applications. Resource optimization will be challenging too since the IoE application requirements might vary from the requirements of existing cellular channels. Smart surveillance/smart automatic driving/smart transportation [5], connected car/fleet management/remote health monitoring/smart metering [4] etc. are some of the potential IoE applications of cellular-based LPWAN technologies. Non-cellular-based LPWAN technologies are more appropriate for IoE applications requiring low data rates with a long communication range, where reliability and mobility are not among the core priorities. Sigfox outdoor localization system [49] and LoRa sailing monitoring system are studied in [50]. While SigFox provides a larger range, LoRa provides more flexibility in terms of data rate as reported in different papers. Also, LoRa has 500 ms one-hop latency while Sigfox has 2s latency [51]. A DASH7 power metering system is analyzed in [52]. Smart cities, smart buildings, smart grids, and oil and gas pipelines are some 
Table 2 - IoE enabling technologies and features

\begin{tabular}{|c|c|c|c|c|c|c|c|}
\hline Technology & $\begin{array}{l}\text { Frequency } \\
\text { Band }\end{array}$ & Range & $\begin{array}{l}\text { Maximum } \\
\text { Date Rate }\end{array}$ & $\begin{array}{l}\text { Channel Band- } \\
\text { width }\end{array}$ & Security & Reliability & Latency \\
\hline LoRa & $\begin{array}{l}868 \mathrm{MHz} \\
915 \mathrm{MHz}\end{array}$ & $15 \mathrm{~km}$ & 50 kbps & $125,250,500 \mathrm{kHz}$ & Low & Low & High \\
\hline SigFox & 915 to $928 \mathrm{MHz}$ & $20 \mathrm{~km}+$ & $100 \mathrm{bps}$ & $100 \mathrm{~Hz}$ & Low & Low & High \\
\hline eMTC & $700-900 \mathrm{MHz}$ & $<15 \mathrm{~km}$ & $1 \mathrm{Mbps}$ & $\begin{array}{l}1.08 \mathrm{MHz} \\
\text { (1.4 MHz carrier } \\
\text { bandwidth) }\end{array}$ & $\begin{array}{l}\text { Medium/ } \\
\text { High }\end{array}$ & $\begin{array}{l}\text { Medium/ } \\
\text { High }\end{array}$ & Low \\
\hline NB-IoT & $700-900 \mathrm{MHz}$ & $<35 \mathrm{~km}$ & $\begin{array}{l}\text { DL: } 170 \mathrm{kbps} \\
\text { UL: } 250 \mathrm{kbps}\end{array}$ & $\begin{array}{l}180 \mathrm{kHz} \\
\text { (200 kHz carrier } \\
\text { bandwidth) }\end{array}$ & $\begin{array}{l}\text { Medium/ } \\
\text { High }\end{array}$ & $\begin{array}{l}\text { Medium/ } \\
\text { High }\end{array}$ & Low \\
\hline $\begin{array}{l}\text { EC-GSM- } \\
\text { IoT }\end{array}$ & $800-900 \mathrm{MHz}$ & $<15 \mathrm{~km}$ & $\begin{array}{l}74 \text { kbps } \\
\text { (GMSK), } 240 \\
\text { kbps (8 PSK) }\end{array}$ & $0.2 \mathrm{MHz}$ & $\begin{array}{l}\text { Medium/ } \\
\text { High }\end{array}$ & $\begin{array}{l}\text { Medium/ } \\
\text { High }\end{array}$ & Low \\
\hline Bluetooth & $2.4 \mathrm{GHz}$ & $50 \mathrm{~m}$ & $2 \mathrm{Mbps}$ & $2 \mathrm{MHz}$ & Low & $\begin{array}{l}\text { Medium/ } \\
\text { High }\end{array}$ & Low \\
\hline ZigBee & $\begin{array}{l}868 \mathrm{MHz}, \\
915 \mathrm{MHz} \text {, and } \\
2.4 \mathrm{GHz}\end{array}$ & $\begin{array}{l}\text { Typically } \\
\text { less than } \\
1 \mathrm{~km}\end{array}$ & $250 \mathrm{kbps}$ & $2 \mathrm{MHz}$ & Low & Low & High \\
\hline $\mathrm{Wi}-\mathrm{Fi}$ & $2.4 \mathrm{GHz}, 5 \mathrm{GHz}$ & $100 \mathrm{~m}$ & $54 \mathrm{Mbps}$ & $22 \mathrm{MHz}$ & $\begin{array}{l}\text { Medium/ } \\
\text { High }\end{array}$ & $\begin{array}{l}\text { Medium/ } \\
\text { High }\end{array}$ & Low \\
\hline
\end{tabular}

potential application domains for non-cellular-based LPWAN technologies.

IoE will also play a major role in industrial automation in the near future $[53,54]$. Most industrial automation applications require high reliability and low latency. Small scale networks such as Wireless Highway Addressable Remote Transducer (WirelessHART), Wireless Interface for Sensors and Actuators (WISA), and Wireless Networks for Industrial Automation for Process Automation (WIAPA), which are based on the IEEE 802.15.4 standard, and the WIAFA [4], which is based on the IEEE 802.11 standards are typically used in industrial automation [30]. However, they do not meet the high scalability and reliability requirements required by many applications. In some industrial applications, the wireless transmission should potentially guarantee the PER around $10^{-9}$ within the transmission delay constraint as low as $10 \mu \mathrm{s}$ [30] which may be difficult for many LPWAN technologies. The current state of the art of different technologies and research studies suggest cellular-based LPWAN technologies are the most suitable candidates for industrial automation applications. URLLC is one of the most important features of the $5 \mathrm{G}$ mobile network. Thus, cellularbased technologies may be able to meet some of the industrial automation application criteria. The typical data size of a packet in an industrial setting is only a few bytes with different update frequency, latency and reliability requirements while the typical communication range is very low. Some valuable insights can be obtained from [4, 34, 55]. ZigBee and Wi-Fi could also be suitable for some industrial applications as well [4].

IoE networks are expected to play a crucial role in improving transportation capability and efficiency. Some communication scenarios for V2X networks are 1) Vehicle to Vehicle (V2V) communications, in which information is exchanged between vehicles; 2) Vehicle to Infrastructure (V2I) communications, which occur between vehicles and Roadside Units (RSUs), traffic lights, and base stations; 3) Vehicle to Pedestrian (V2P) communications, in which vehicles communicate with people who are along the side of the road; and 4) Vehicle to Network (V2N), where the vehicles connect to an entity in the networks e.g., a backend server or a traffic information system [30]. However, the requirements on latency and reliability are very high for V2X networks. Some basic requirements for V2X communication networks are low latency, high reliability, high throughput, interference-robust, communication range and mobility support. It is expected that $5 \mathrm{G}$ cellular networks will play an important role in this application domain.

Currently, most of the LPWAN technologies use a star topology and rely on wired infrastructure (e.g., cellular LPWANs) or Internet (e.g., LoRaWAN) to integrate multiple networks to cover large areas. The adoption of LPWAN technologies in rural and remote area applications such as agricultural IoE and industrial IoE (e.g., for oil/gas fields) that may cover large areas is challenging. Some technologies for achieving last-mile connectivity have been discussed in [56]. Cellular networks can be an efficient last-mile solution for rural areas due to significant cellular penetration in many rural areas across the world. Although WiFi is a mature technology, the IEEE 802.11 MAC protocol gives poor end-to-end performance for long-range communication. Femtocell, which uses a small low-power cellular base station, can be used to provide cost-effective cellular connectivity within its 
Table 3 - Possible technologies for some IoE application scenarios

\begin{tabular}{|c|c|c|}
\hline Application & Application Requirements & Possible Technologies \\
\hline Structural Health (Smart City) & $\begin{array}{l}\text { Tolerable delay: } 30 \text { min, update frequency: } \\
10 \text { min, data rate: low }\end{array}$ & $\begin{array}{l}\text { LoRaWAN, SigFox, LTE, NB- } \\
\text { IoT, ZigBee }\end{array}$ \\
\hline Waste Management (Smart City) & $\begin{array}{l}\text { Tolerable delay: } 30 \mathrm{~min} \text {, update frequency: } \\
1 \text { hour, data rate: low }\end{array}$ & $\begin{array}{l}\text { LoRaWAN, SigFox, LTE, NB- } \\
\text { IoT }\end{array}$ \\
\hline Video Surveillance (Smart City) & $\begin{array}{l}\text { Tolerable delay: seconds, update frequency: } \\
\text { real-time, data rate: high, netwaork coverage: } \\
\text { large/small }\end{array}$ & LTE, NB-IoT, WiFi \\
\hline $\begin{array}{l}\text { Air Quality Monitoring (Smart } \\
\text { Home) }\end{array}$ & $\begin{array}{l}\text { Tolerable delay: } 5 \mathrm{~min} \text {, update frequency: } \\
30 \mathrm{~min} \text {, data rate: low }\end{array}$ & Wi-Fi, Bluetooth, NB-IoT \\
\hline $\begin{array}{l}\text { Patients Healthcare Delivery and } \\
\text { Monitoring (Healthcare) }\end{array}$ & $\begin{array}{l}\text { Tolerable delay: seconds, update frequency: } \\
1 \text { report per hour/day, data rate: high } \\
\text { security: high, reliability: high }\end{array}$ & Bluetooth, LTE, NB-IoT \\
\hline $\begin{array}{l}\text { Real-time Emergency Response } \\
\text { and Remote Diagnostics (Health- } \\
\text { care) }\end{array}$ & $\begin{array}{l}\text { Tolerable delay: seconds, update frequency: } \\
\text { ad-hoc emergency communication, data rate: } \\
\text { high security: high, reliability: high }\end{array}$ & Bluetooth, LTE, NB-IoT \\
\hline Smart Grids (Industrial) & $\begin{array}{l}\text { Tolerable delay: } 3 \text { to } 20 \mathrm{~ms} \text {, update frequency: } \\
10 \text { to } 100 \mathrm{~ms} \text {, reliability: } 10^{-6} \text { PLR, network cov- } \\
\text { erage: a few meter to kilometers }\end{array}$ & $\begin{array}{l}\text { WiFi, ZigBee, LTE, WiMAX, } \\
\text { NB-IoT }\end{array}$ \\
\hline Road Safety Highway (Smart City) & $\begin{array}{l}\text { Tolerable delay: } 10 \text { to } 100 \mathrm{~ms} \text {, update fre- } \\
\text { quency: } 100 \mathrm{~ms} \text {, reliability: } 10^{-3} \text { to } 10^{-5} \text { PLR, } \\
\text { network coverage: } 2000 \mathrm{~m}\end{array}$ & LTE, NB-IoT \\
\hline Factory Automation (Industrial) & $\begin{array}{l}\text { Tolerable delay: } 0.25 \text { to } 10 \mathrm{~ms} \text {, update fre- } \\
\text { quency: } 0.5 \text { to } 50 \mathrm{~ms} \text {, reliability: } 10^{-9} \text { PLR, net- } \\
\text { work coverage: } 50 \text { to } 100 \mathrm{~m}\end{array}$ & LTE, NB-IoT, WiFi \\
\hline Manufacturing Cell (Industrial) & $\begin{array}{l}\text { Tolerable delay: } 5 \mathrm{~ms} \text {, update frequency: } 50 \mathrm{~ms} \text {, } \\
\text { reliability: } 10^{-9} \text { PLR, network coverage: } 50 \text { to } \\
100 \mathrm{~m}\end{array}$ & LTE, NB-IoT, WiFi \\
\hline Process Automation (Industrial) & $\begin{array}{l}\text { Tolerable delay: } 50 \text { to } 100 \mathrm{~ms} \text {, update fre- } \\
\text { quency: } 100 \text { to } 5000 \mathrm{~ms} \text {, reliability: } 10^{-3} \text { to } 10^{-4} \\
\text { PLR, network coverage: } 100 \text { to } 500 \mathrm{~m}\end{array}$ & LTE, NB-IoT \\
\hline
\end{tabular}

coverage range. High user mobility and extended battery life of mobile terminals can be achieved using LTE. WiMAX supports broadband applications as well as providing large coverage, and deployment of a WiMAX network is much cheaper than the deployment of an LTE network for last-mile connectivity. Cognitive radio technologies can achieve large coverage with non-LoS links in lastmile connectivity in rural areas utilizing unused licensed spectrum.

The application of IoE promises smart, innovative and comfortable medical services to the patients and/or individuals needing healthcare, and furnishes their class of life through easing emergency medical support, security and continuous care [57]. The prospective applications of IoE in medical sectors include health monitoring using wearable devices that measure the physical activities/behaviour [58], supporting health-related information for regular patient care, and networking through devices for clinical care with issues of an unvarying electrocardiogram, blood oxygen and blood pressure [59]. IoE can lead to constructing big data on a particular health issue and can play a pivotal role in the further progress of IoE through the analysis and application of big data. Also, IoE has the prospect of on-time medical assistance by connecting the network to traffic and hospital administration in case of an accident. Moreover, IoE also supports the electronic reporting of patients' mobility (i.e. contact tracing) to ensure homecare. IoE-coupled smart wearable devices/systems have been reported for monitoring cardiovascular disease, Chronic Obstructive Pulmonary Disease (COPD), Parkinson's disease, pregnancy and cognitive disorder. Usually, the acquired data (biomarkers such as ECG, respiratory rate, body temperature, EMG muscle activity, gait and others) using sensory devices are transmitted to the Healthcare Organization (HCO) using the intermediate concentrators and platforms connected with short-range radio such as Zigbee or low-power Bluetooth under the governance of a smartphone's WiFi or cellular data connection [60].

Some IoE applications, typical requirements and possible technologies are shown in Table 3. Wearables and smart metering are two potential IoE application areas. The networking technologies used in these technologies are discussed in the following subsections. 


\subsection{Wearables in healthcare}

In today's digital world the term "wearable" refers to accessories such as a smartwatch on a business executive's wrist, a head-mounted display worn by an immersive gamer, a tiny sensor on a cyclist's helmet, or a smart garment a runner uses to track and monitor his steps [61]. The ability of sensing comes from the embedded sensors in wearables. The functional attributes such as multi-functionality, configurability, responsiveness and bandwidth depend on the nature of an application. Currently, two industry giants, Apple and Google dominate the wearable technology market by-products released [62]. The seamless integration of wearables in healthcare settings will have to ensure compatibility with existing wireless technologies and established operational protocols in these settings. Sensor Platform for Healthcare in Residential Environment (SPHERE) is a multi-modal platform of non-medical sensors for behaviour monitoring in residential environments that utilize inherently costefficient and scalable IoE technologies $[63,64]$. The original health evidence is collected from the physiological signals of a human body using diverse biosensors. These biosensors can be deployed in an implantable (in-body), wearable (on-body), portable (off-body) or environmental modality. The home environment and the resident interaction with the environment are monitored in a Home (SH) by a system of pervasive information and communication technologies consisting of sensor systems.

Enabling the sensing platform for remote monitoring requires networking technologies to provide ubiquitous network connectivity between residents and clinicians. LTE and Bluetooth are possible networking solutions for medical sensors as the application requires low latency, high reliability and low capacity [65]. Energy-efficient, IP-enabled sensing networks can allow access to existing Internet infrastructures removing the need for translation gateways or proxies in hardware and software. It will improve the user experience and require less maintenance effort. Although WiFi has the significant advantage of being Internet Protocol (IP) enabled, the hardware used in WiFi connectivity consumes relatively more power and therefore, less suitable for long-term deployments of an application that utilizes battery-powered sensor nodes. 6LoWPAN has better support for multi-hop mesh and thus, it was selected for the environmental sensor network and data forwarding in SPHERE [63]. On the other hand, BLE was chosen for collecting the data from the wearable nodes for being more convenient. SPHERE uses IPv6 on top of the IEEE 802.15.4 TSCH protocol to provide time synchronization to the network and ensure time-stamping all of sensor data with high accuracy. ZigBee was used in the first version of the SPHERE. However, ZigBee uses a single channel at a time and does not have time slots. WiGig products based on IEEE 802.11ad may replace Bluetooth and $\mathrm{WiFi}$ at some point in future for applications with high throughput requirements as Bluetooth and WiFi have very limited scaling capability.

\subsection{Smart metering}

Advanced Metering Infrastructure (AMI) is an integral part of Smart Grids (SGs) and smart metering is one of the most promising applications of IoE. AMI, besides enabling accurate consumer billing in the presence of dynamic pricing and improving efficiency and reliability of electricity distribution in the presence of distributed generation, will be used in water and gas utility distribution networks in smart cities as an application of IoE. Renewable energy producers and mobile energy storage can be linked and utilized by SGs' infrastructure. AMI communication networks can be divided into Home Area Networks (HANs), Neighborhood Area Networks (NANs) or Meter Local Area Networks (MLAN) and Wide Area Networks (WANs) $[66,67]$. Connections among distributed energy resources, GWs, Electric Vehicles (EVs), Smart Meters (SMs), etc. are provided by the HANs. SMs that need to send their data to the corresponding data concentrator are facilitated by the NANs or MLAN. Appliances such as entertainment systems, lighting systems, energy storage and EVs constitute HANs and SMs act as home GWs that link the HANs with the NANs [68]. Connections between some data concentrators and the central system are provided by WANs.

The choice of a suitable technology in AMI depends on application requirements such as security, privacy, bandwidth, latency, reliability, energy efficiency etc. Power Line Communications (PLC) and wireless communications are widely used in SGs as the overall system reliability can be enhanced by exploiting the diversity achieved from the simultaneous transmission of the same signal over power lines and wireless links. Wireless Sensor Networks (WSNs) are attractive solutions for AMI because of their low-cost deployment and multiple functionalities. However, one of the challenging tasks for WSNs is to ensure QoS requirements for AMI applications. Typically, SMs are connected to the Distribution System Operators' (DSO) backend system in two ways: 1) a concentrator gathers the data from the SMs in its neighbourhood using Wi-Fi or PLC connections and then relays it using cellular or a wired connection to the DSO backend, or 2) Each SM sends data to the DSO backend using a cellular network [69]. IEEE 802.15 .4 (e.g., ZigBee and Zwave), IEEE 802.11 (WiFi) are some of the technologies used in HANs [66]. Although PLC has been the primary choice for communication between the SMs and data concentrators, wireless mesh networks in AMI have been proposed and deployed widely. The use of LTE as a NAN technology was discussed in [68]. Some of the potential WAN technologies are IEEE 802.16 (i.e., WiMAX), IEEE 802.20 (MobileFi), PLC, IEEE 802.11 (WiFi) and IEEE 802.15.4 (ZigBee) [66]. LoRaWAN can be used in applications with relaxed QoS requirements such as latency tolerant services of a Power Wireless Private Network (PWPN) [70]. 


\section{CONCLUSIONS}

The paper discussed IoE application requirements such as latency, energy efficiency, data rate, reliability, security and communication range. Features, advantages and disadvantages of short-range, cellular and non-cellularbased IoE enabling technologies are presented as well. It is evident from the discussion that choosing a particular IoE enabling technology depends on the specific application. It is also possible that specific application requirements are met through more than one existing technology. However, it is very likely that among the potential technologies, one technology performs better than others with a priority list of key network performance indicators. Understanding the application requirements and technological features will play a key role in determining the most suitable IoE enabling technology for a particular application.

\section{ACKNOWLEDGEMENT}

This project was supported in part by the University Research Center, Shahjalal University of Science and Technology under the research grant: AS/2020/1/31.

\section{REFERENCES}

[1] Al-Fuqaha, A., Guizani, M., Mohammadi, M., Aledhari, M., \& Ayyash, M. (2015), Internet of things: A survey on enabling technologies, protocols, and applications, IEEE communications surveys \& tutorials, 17(4), 2347-2376.

[2] Saxena, N., Roy, A., Sahu, B. J., \& Kim, H. (2017), Efficient IoT gateway over 5G wireless: A new design with prototype and implementation results, IEEE Communications Magazine, 55(2), 97-105.

[3] Dhillon, H. S., Huang, H., \& Viswanathan, H. (2017), Wide-area wireless communication challenges for the Internet of Things, IEEE Communications Magazine, 55(2), 168-174.

[4] Stankovic, J. A. (2014), Research directions for the internet of things, IEEE Internet of Things Journal, 1(1), 3-9.

[5] Akpakwu, G. A., Silva, B. J., Hancke, G. P., \& AbuMahfouz, A. M. (2017), A survey on 5G networks for the Internet of Things: Communication technologies and challenges, IEEE access, 6, 3619-3647.

[6] Stefanizzi, M. L., Mottola, L., Mainetti, L., \& Patrono, L. (2017), COIN: Opening the internet of things to people's mobile devices, IEEE Communications Magazine, 55(2), 20-26.

[7] Chen, S., Liu, T., Gao, F., Ji, J., Xu, Z., Qian, B., \& Guan, X. (2017), Butler, not servant: A human-centric smart home energy management system, IEEE Communications Magazine, 55(2), 27-33.
[8] Martella, C., Cattani, M., \& Van Steen, M. (2017), Exploiting density to track human behavior in crowded environments, IEEE Communications Magazine, $55(2), 48-54$.

[9] Jiang, J., Han, G., Zhu, C., Chan, S., \& Rodrigues, J. J. (2017), A trust cloud model for underwater wireless sensor networks, IEEE Communications Magazine, 55(3), 110-116.

[10] https://www.statista.com/statistics/764026/numberof-iot-devices-in-use-worldwide/ (Accessed: 27 Feb, 2021)

[11] Yao, Y., Zhu, Z., Huang, S., Yue, X., Pan, C., \& Li, X. (2019), Energy Efficiency Characterization in Heterogeneous IoT System With UAV Swarms Based on Wireless Power Transfer, IEEE Access, 8, 967-979.

[12] Paul, B. (2020), A Novel Mathematical Model to Evaluate the Impact of Packet Retransmissions in LoRaWAN, IEEE Sensors Letters, 4(5), 1-4.

[13] Wu, Q., Chen, W., Ng, D. W. K., \& Schober, R. (2018), Spectral and energy-efficient wireless powered IoT networks: NOMA or TDMA?, IEEE Transactions on Vehicular Technology, 67(7), 6663-6667.

[14] Paul, B. (2020), A Novel Energy-Efficient Routing Scheme for LoRa Networks, IEEE Sensors Journal, 20(15), 8858-8866.

[15] Nguyen, T. D., Khan, J. Y., \& Ngo, D. T. (2018), A distributed energy-harvesting-aware routing algorithm for heterogeneous IoT networks, IEEE Transactions on Green Communications and Networking, 2(4), 1115-1127.

[16] Sen, S., Koo, J., \& Bagchi, S. (2018), TRIFECTA: security, energy efficiency, and communication capacity comparison for wireless IoT devices, IEEE Internet Computing, 22(1), 74-81.

[17] Alzahrani, B., \& Ejaz, W. (2018), Resource management for cognitive IoT systems with RF energy harvesting in smart cities, IEEE Access, 6, 62717-62727.

[18] Liu, X., Li, Z., Zhao, N., Meng, W., Gui, G., Chen, Y., \& Adachi, F. (2018), Transceiver design and multihop D2D for UAV IoT coverage in disasters, IEEE Internet of Things Journal, 6(2), 1803-1815.

[19] Ikpehai, A., Adebisi, B., Rabie, K. M., Anoh, K., Ande, R. E., Hammoudeh, M., ... \& Mbanaso, U. M. (2018), Low-power wide area network technologies for internet-of-things: A comparative review, IEEE Internet of Things Journal, 6(2), 2225-2240.

[20] Neshenko, N., Bou-Harb, E., Crichigno, J., Kaddoum, G., \& Ghani, N. (2019), Demystifying IoT security: an exhaustive survey on IoT vulnerabilities and a first empirical look on internet-scale IoT exploitations, IEEE Communications Surveys \& Tutorials, 21(3), 2702-2733. 
[21] Iqbal, W., Abbas, H., Daneshmand, M., Rauf, B., \& Bangash, Y. A. (2020), An In-Depth Analysis of IoT Security Requirements, Challenges, and Their Countermeasures via Software-Defined Security, IEEE Internet of Things Journal, 7(10), 10250-10276.

[22] Hassija, V., Chamola, V., Saxena, V., Jain, D., Goyal, P., \& Sikdar, B. (2019), A survey on IoT security: application areas, security threats, and solution architectures, IEEE Access, 7, 82721-82743.

[23] Giust, F., Cominardi, L., \& Bernardos, C. J. (2015), Distributed mobility management for future $5 \mathrm{G}$ networks: overview and analysis of existing approaches, IEEE Communications Magazine, 53(1), 142-149.

[24] Shin, D., Yun, K., Kim, J., Astillo, P. V., Kim, J. N., \& You, I. (2019), A security protocol for route optimization in DMM-based smart home IoT networks, IEEE Access, 7, 142531-142550.

[25] Mikhaylov, K., Petaejaejaervi, J., \& Haenninen, T. (2016, May), Analysis of capacity and scalability of the LoRa low power wide area network technology, In European Wireless 2016; 22th European Wireless Conference; Proceedings of (pp. 1-6). VDE.

[26] Alsohaily, A., Sousa, E., Tenenbaum, A. J., \& Maljevic, I. (2017, October), LoRaWAN radio interface analysis for North American frequency band operation, In Personal, Indoor, and Mobile Radio Communications (PIMRC), 2017 IEEE 28th Annual International Symposium on (pp. 1-6). IEEE.

[27] Reynders, B., Wang, Q., Tuset-Peiro, P., Vilajosana, X., \& Pollin, S. (2018), Improving Reliability and Scalability of LoRaWANs Through Lightweight Scheduling, IEEE Internet of Things Journal.

[28] Rahman, M., \& Saifullah, A. (2020), Integrating lowpower wide-area networks for enhanced scalability and extended coverage, IEEE/ACM Transactions on Networking, 28(1), 413-426.

[29] Adame, T., Bel, A., \& Bellalta, B. (2019), Increasing LPWAN scalability by means of concurrent multiband IoT technologies: an industry 4.0 use case, IEEE Access, 7, 46990-47010.

[30] Ma, Z., Xiao, M., Xiao, Y., Pang, Z., Poor, H. V., \& Vucetic, B. (2019), High-reliability and low-latency wireless communication for internet of things: challenges, fundamentals, and enabling technologies, IEEE Internet of Things Journal, 6(5), 7946-7970.

[31] Xing, L. (2020), Reliability in Internet of Things: Current status and future perspectives, IEEE Internet of Things Journal, 7(8), 6704-6721.
[32] Bagchi, S., Abdelzaher, T. F., Govindan, R., Shenoy, P., Atrey, A., Ghosh, P., \& Xu, R. (2020), New Frontiers in IoT: Networking, Systems, Reliability, and Security Challenges, IEEE Internet of Things Journal, 7(12), 11330-11346.

[33] Jia, G., Zhu, Y., Li, Y., Zhu, Z., \& Zhou, L. (2019), Analysis of the Effect of the Reliability of the NB-Iot Network on the Intelligent System, IEEE Access, 7, 112809-112820.

[34] Schulz, P., Matthe, M., Klessig, H., Simsek, M., Fettweis, G., Ansari, J., \& Puschmann, A. (2017), Latency critical IoT applications in 5G: Perspective on the design of radio interface and network architecture, IEEE Communications Magazine, 55(2), 70-78.

[35] Zhang, C., Sun, X., Zhang, J., Wang, X., Jin, S., \& Zhu, H. (2019), Throughput optimization with delay guarantee for massive random access of M2M communications in industrial IoT, IEEE Internet of Things Journal, 6(6), 10077-10092.

[36] Moussa, H. G., \& Zhuang, W. (2019), Energy-and delay-aware two-hop NOMA-enabled massive cellular IoT communications, IEEE Internet of Things Journal, 7(1), 558-569.

[37] Deng, Y., Chen, Z., Yao, X., Hassan, S., \& Ibrahim, A. M. (2019), Parallel offloading in green and sustainable mobile edge computing for delay-constrained IoT system, IEEE Transactions on Vehicular Technology, 68(12), 12202-12214.

[38] Yi, C., \& Cai, J. (2018), A truthful mechanism for scheduling delay-constrained wireless transmissions in IoT-based healthcare networks, IEEE Transactions on Wireless Communications, 18(2), 912-925.

[39] Li, T., Ota, K., Wang, T., Li, X., Cai, Z., \& Liu, A. (2019), Optimizing the coverage via the UAVs with lower costs for information-centric Internet of Things, IEEE Access, 7, 15292-15309.

[40] Kwon, T., Choi, S. W., \& Shin, Y. H. (2019), A comprehensive design framework for network-wide cost reduction in random access-based wireless IoT networks, IEEE Communications Letters, 23(9), 15761580 .

[41] Agiwal, M., Roy, A., \& Saxena, N. (2016), Next generation 5G wireless networks: A comprehensive survey, IEEE Communications Surveys \& Tutorials, 18(3), 1617-1655.

[42] Buzzi, S., Chih-Lin, I., Klein, T. E., Poor, H. V., Yang, C., \& Zappone, A. (2016), A survey of energy-efficient techniques for $5 \mathrm{G}$ networks and challenges ahead, IEEE Journal on Selected Areas in Communications, 34(4), 697-709. 
[43] Paul, B. (2019). Impacts of Multi-Hop Routing and Channel/Transmission Configuration Planning on LoRa Networks (MSc dissertation, University of Saskatchewan).

[44] Raza, U., Kulkarni, P., \& Sooriyabandara, M. (2017), Low power wide area networks: An overview, IEEE Communications Surveys \& Tutorials, 19(2), 855873.

[45] Poursafar, N., Alahi, M. E. E., \& Mukhopadhyay, S. (2017, December), Long-range wireless technologies for IoT applications: A review, In Sensing Technology (ICST), 2017 Eleventh International Conference on (pp. 1-6). IEEE.

[46] Alapetite, A., \& Hansen, J. P. (2016, December), Dynamic Bluetooth beacons for people with disabilities, In Internet of Things (WF-IoT), 2016 IEEE 3rd World Forum on (pp. 36-41). IEEE.

[47] Vikram, N., Harish, K. S., Nihaal, M. S., Umesh, R., Shetty, A., \& Kumar, A. (2017, January), A low cost home automation system using wi-fi based wireless sensor network incorporating Internet of Things (IoT), In Advance Computing Conference (IACC), 2017 IEEE 7th International (pp. 174-178). IEEE.

[48] Karthikeyan, S., Rani, G. S., Sridevi, M., \& Bhuvaneswari, P. T. V. (2017, May), IoT enabled waste management system using ZigBee network, In Recent Trends in Electronics, Information \& Communication Technology (RTEICT), 2017 2nd IEEE International Conference on (pp. 2182-2187). IEEE.

[49] Ribeiro, G. G., de Lima, L. F., Oliveira, L., Rodrigues, J. J., Marins, C. N., \& Marcondes, G. A. (2018, June), An Outdoor Localization System Based on SigFox, In 2018 IEEE 87th Vehicular Technology Conference (VTC Spring) (pp. 1-5). IEEE.

[50] Li, L., Ren, J., \& Zhu, Q. (2017, February), On the application of LoRa LPWAN technology in Sailing Monitoring System, In Wireless On-demand Network Systems and Services (WONS), 2017 13th Annual Conference on (pp. 77-80). IEEE.

[51] Morin, E., Maman, M., Guizzetti, R., \& Duda, A. (2017), Comparison of the device lifetime in wireless networks for the internet of things, IEEE Access, 5, 7097-7114.

[52] Cetinkaya, O., \& Akan, O. B. (2015, January), A DASH7-based power metering system, In Consumer Communications and Networking Conference (CCNC), 2015 12th Annual IEEE(pp. 406-411). IEEE.

[53] Wollschlaeger, M., Sauter, T., \& Jasperneite, J. (2017), The future of industrial communication: Automation networks in the era of the internet of things and industry 4.0., IEEE Industrial Electronics Magazine, 11(1), 17-27.
[54] Delsing, J. (2017), Local cloud internet of things automation: Technology and business model features of distributed internet of things automation solutions, IEEE Industrial Electronics Magazine, 11(4), 8-21.

[55] Lennvall, T., Gidlund, M., \& Åkerberg, J. (2017, September), Challenges when bringing IoT into industrial automation, In AFRICON, 2017 IEEE (pp. 905-910). IEEE.

[56] Nandi, S., Thota, S., Nag, A., Divyasukhananda, S., Goswami, P., Aravindakshan, A., ... \& Mukherjee, B. (2016), Computing for rural empowerment: enabled by last-mile telecommunications, IEEE Communications Magazine, 54(6), 102-109.

[57] Gatouillat, A., Badr, Y., Massot, B., \& Sejdić, E. (2018), Internet of medical things: A review of recent contributions dealing with cyber-physical systems in medicine, IEEE Internet of Things Journal, 5(5), 38103822.

[58] Son, D., Lee, J., Qiao, S., Ghaffari, R., Kim, J., Lee, J. E., ... \& Kim, D. H. (2014), Multifunctional wearable devices for diagnosis and therapy of movement disorders, Nature nanotechnology, 9(5), 397.

[59] Paradiso, R., Loriga, G., \& Taccini, N. (2005), A wearable health care system based on knitted integrated sensors, IEEE transactions on Information Technology in biomedicine, 9(3), 337-344.

[60] Hu, F., Xie, D., \& Shen, S. (2013, August). Hu, F., Xie, D., \& Shen, S. (2013, August), On the application of the internet of things in the field of medical and health care, In 2013 IEEE international conference on green computing and communications and IEEE Internet of Things and IEEE cyber, physical and social computing (pp. 2053-2058). IEEE.

[61] Park, S., \& Jayaraman, S. (2021), Wearables: Fundamentals, advancements, and a roadmap for the future, In Wearable sensors (pp. 3-27). Academic Press.

[62] Pyattaev, A., Johnsson, K., Andreev, S., \& Koucheryavy, Y. (2015), Communication challenges in highdensity deployments of wearable wireless devices, IEEE Wireless Communications, 22(1), 12-18.

[63] Elsts, A., Fafoutis, X., Woznowski, P., Tonkin, E., Oikonomou, G., Piechocki, R., \& Craddock, I. (2018), Enabling healthcare in smart homes: the SPHERE IoT network infrastructure, IEEE Communications Magazine, 56(12), 164-170.

[64] Zhu, N., Diethe, T., Camplani, M., Tao, L., Burrows, A., Twomey, N., ... \& Craddock, I. (2015), Bridging ehealth and the internet of things: The sphere project, IEEE Intelligent Systems, 30(4), 39-46. 
[65] Sun, H., Zhang, Z., Hu, R. Q., \& Qian, Y. (2018), Wearable communications in $5 \mathrm{G}$ : challenges and enabling technologies, IEEE vehicular technology magazine, 13(3), 100-109.

[66] Kumar, P., Lin, Y., Bai, G., Paverd, A., Dong, J. S., \& Martin, A. (2019), Smart grid metering networks: A survey on security, privacy and open research issues, IEEE Communications Surveys \& Tutorials, 21(3), 2886-2927.

[67] Ye, F., Qian, Y., Hu, R. Q., \& Das, S. K. (2015), Reliable energy-efficient uplink transmission for neighborhood area networks in smart grid, IEEE Transactions on Smart Grid, 6(5), 2179-2188.

[68] Anjana, K. R., \& Shaji, R. S. (2018), A review on the features and technologies for energy efficiency of smart grid, International Journal of Energy Research, 42(3), 936-952.

[69] Bou-Harb, E., Fachkha, C., Pourzandi, M., Debbabi, M., \& Assi, C. (2013), Communication security for smart grid distribution networks, IEEE Communications Magazine, 51(1), 42-49.

[70] Bao, L., Wei, L., Jiang, C., Miao, W., Guo, B., Li, W., ... \& Zou, J. (2018), Coverage analysis on NB-IoT and LoRa in power wireless private network, Procedia computer science, 131, 1032-1038.

\section{AUTHORS}

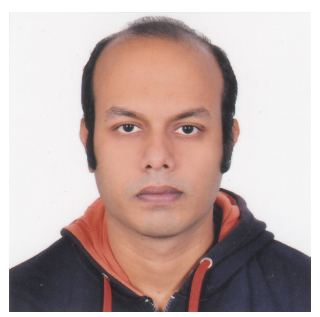

Biswajit Paul completed his BSc in Electronics and Telecommunication Engineering from North South University, Bangladesh with the distinction of Summa Cum Laude and MSc from University of Saskatchewan, Canada. He started his career as a Lecturer at Leading University and later joined Shahjalal University of Science and Technology (SUST). Currently he is an Associate Professor in the Department of Electrical and Electronic Engineering at SUST. He was the Founder Chairman of IEEE Student Branch, NSU. So far, he has published a few referred international journal and conference papers. He also serves as a reviewer for some prestigious international journals.

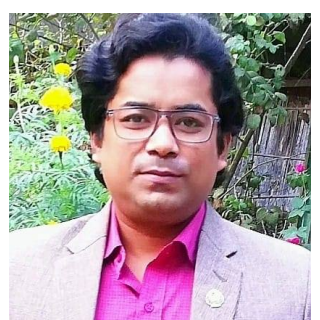

\section{Gokul Chandra Biswas} achieved his B.Sc. in agriculture and MS in biotechnology from the Bangladesh Agricultural University, Bangladesh in 2007 and 2009 respectively. He received his Ph.D. in Nano-Science and Nano-Technology in 2017 from the University of Tsukuba, Japan. In 2010, He joined Bangladesh Agricultural Research Institute as scientist. In 2011, he moved as lecturer to Shahjalal University of Science and Technology (SUST), Bangladesh. Since 2017, he is an associate professor of the Department of
Genetic Engineering and Biotechnology, SUST. His research interest is on the point-of-care diagnostics, micro-total-analysis-system ( $\mu \mathrm{TAS})$, biomedical engineering and evidence-based disease surveillance. He has published many peer-reviewed articles in reputed international journals. He became one of the founding members of Bangladesh Nano Society in 2020. He has also membership for several professional scientific communities of life science research arenas.

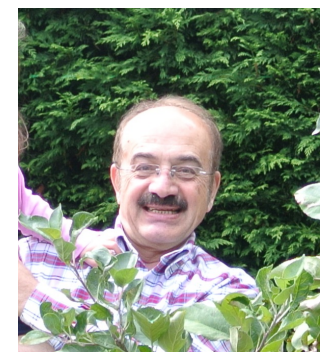

Habib F. Rashvand, CEng, LIEEE received his distinguished engineering $\mathrm{BE}$ and Postgrad Diploma qualifications from the University of Tehran in 1970 and 1971. Then, selected for training to head a major research and development operation visited Japan for representing the University and Iranian PTT in a two-years association with NTT, KTT and other Japanese industries; and, headed an international project for building the Telecom Research Centre (ITRC) as a distinct national resources of the country. His Doctorate of Philosophy from the University of Kent in 1980 shows his appetite for his contributions to the new world of data communications and the Internet with his industrial presence in high-speed modems in the 1980s and in the 1990s in mobile and wireless technologies until his professorship on 'Networks, Systems \& Protocols' granted in 2001 by the German Ministry of Education. His rich blend of 30 continuous years of industrial and academic research and development involving international industries including Racal, Vodafone, Nokia and Cable \& Wireless at various senior positions worked and collaborated with a wide range of academies including University of Tehran, University of Zambia, Portsmouth University, Southampton University, Warwick \& Coventry Universities, Open University and Magdeburg German Universities. He has direct consultation experience as Editor-in-Chief(s) and Guest Editor(s) for IEE, IET and IEEE for editorial issues and research journals for over 15 years. He has presented many prestigious keynotes and and has been invited as a guest speaker, and has managed well over 100 cooperative projects, five books and over 100 research papers and book chapters. His technical books are titled: Distributed Sensor Systems (Wiley 2012), Using Cross-Layer Techniques for Communication Systems (IGI Global 2012ed), Dynamic Ad Hoc Networks (IET 2013ed), Wireless Sensor Systems (Wiley 2017ed), and Design Solutions for Wireless Sensor Networks in Extreme Environments (Artech House 2019). Since 2004, seeking for a technology oriented innovative ICT solutions paradigm for a better future of humanity in association with University of Warwick, heading a special operation as the Director of Advanced Communication Systems involving global academics, innovative industries and professional institutions, he has dedicated his time to help build a sustainable future global village. 